\title{
Factors Related to Failure of Conservative Treatment in Volar Plate Avulsion Fractures of the Proximal Interphalangeal Joint
}

\author{
Sanglim Lee, MD, Seung Jin Jang, MD*, Suk Ha Jeon, $\mathrm{MD}^{*}$ \\ Department of Orthopedic Surgery, Inje University Sanggye Paik Hospital, Seoul, \\ ${ }^{*}$ Department of Orthopaedic Surgery, National Medical Center, Seoul, Korea
}

Background: Volar plate avulsion fractures of the proximal interphalangeal (PIP) joint are a common hand injury and have been treated conservatively with favorable results. We assumed that conservative treatment of volar plate avulsion fractures of the PIP joint would be unsuccessful if the fracture fragment, even if small, was much displaced or rotated and that delayed excision of the avulsion fractures would result in good outcomes. We report clinical and radiological outcomes of conservative treatment of volar plate avulsion fractures of the PIP joint and risk factors for failure of conservative treatment.

Methods: We retrospectively reviewed the clinical and radiological outcomes of 88 volar plate avulsion fractures (85 patients) treated conservatively at first. In 18 of these fractures, delayed excision of the fracture fragment was required after an average of 75 days of conservative treatment for limited motion or pain of the joint. We compared parameters between failed cases and successful cases after conservative treatment.

Results: Compared to the successful cases, the failed cases had a higher prevalence of joint dislocation at the time of injury and greater pain, larger flexion contracture, and less further flexion after conservative treatment. The shape, comminution, and size of the fracture fragments were not related with the need for operation, but the operative cases had greater displacement and rotation of the fracture fragments than the conservative cases. After fragment excision, postoperative protection of the joint was not necessary, pain was reduced, and the mean range of motion increased.

Conclusions: The presence of joint dislocation and greater displacement and rotation of the fragments may be associated with the failure of conservative treatment of volar plate avulsion fractures. Failed cases after conservative treatment could be resolved by delayed fragment excision with favorable results. Therefore, it might be appropriate to consider conservative treatment at first in almost all volar plate avulsion fractures of stable PIP joints.

Keywords: Finger, Volar plate, Avulsion fracture, Conservative treatment, Excision

Volar plate avulsion fractures of the proximal interphalangeal (PIP) joint of the finger have been treated conservatively with favorable results. ${ }^{1-3)}$ Gaine et al. ${ }^{1)}$ reported an excellent or good outcome in $98 \%$ of 162 fractures, but

Received October 25, 2019; Accepted January 13, 2020

Correspondence to: Suk Ha Jeon, MD

Department of Orthopaedic Surgery, National Medical Center, 245 Eulji-

ro, Jung-gu, Seoul 04564, Korea

Tel: +82-2-2260-7198, Fax: +82-2-2278-9570

E-mail: lotusjeon@gmail.com they excluded cases with joint dislocation and joint instability. Phair et al. ${ }^{2)}$ reported good results in $96 \%$ of 74 volar avulsion fractures without radiological subluxation where the fragment size was $2 \times 2 \mathrm{~mm}$ or less. They suggested that poor results would occur in fracture-dislocations and fractures with lateral instability. Adi et al. ${ }^{3)}$ conservatively treated 75 volar plate injuries including 23 volar plate avulsion fractures with favorable results and showed that the prognosis of conservative treatment was not related to the presence of an avulsion fracture. These studies support the notion that when there is no joint dislocation or lateral 
Lee et al. Conservative Treatment of Volar Plate Avulsion Fractures of Proximal Interphalangeal Joint

Clinics in Orthopedic Surgery • Vol. 12, No. 3, $2020 \bullet$ www.ecios.org

instability and the fracture fragment is small, volar plate avulsion fractures can be successfully treated by conservative management.

However, if large fracture fragments are displaced or rotated, internal fixation is recommended to prevent joint incongruity. ${ }^{4}$ In some cases, even small fragments should be excised to relieve the pain and improve range of motion (ROM) after conservative treatment since the actual size of the fragments is larger than that observed on radiographs and the fragments are rotated. ${ }^{5)}$

We have usually treated volar plate avulsion fractures of the PIP joint conservatively unless the fracture fragment was larger than $30 \%$ of the articular surface. We assumed that conservative treatment of volar plate avulsion fractures of the PIP joint would be unsuccessful if the fracture fragment, even if small, was severely displaced or rotated and that delayed excision of avulsion fractures would also result in good outcomes. To test these hypotheses, we evaluated the clinical outcomes of conservative treatment of volar plate avulsion fractures of the PIP joint and investigated risk factors for failure of conservative treatment.

\section{METHODS}

We retrospectively reviewed the medical records and radiological results of patients with volar plate avulsion fractures of the PIP joint of the finger who presented to our institution between September 2009 and December 2016. Inclusion criteria were volar plate avulsion fractures of the PIP joint of the finger treated conservatively at first. Stable PIP joints after dislocation were included if there was no definite evidence of dorsal subluxation or instability after reduction. The prevalence of a concurrent joint dislocation at the time of injury was noted in each case. Even joints with grade III coronal instability in the varus or valgus stress test were included. Fractures in any displacement with no articular step-off were included. Avulsion fractures larger than $30 \%$ of the articular surface or fractures with an articular step-off more than $2 \mathrm{~mm}$ were considered as a primary indication for operative treatment and thus were excluded from the study. Joints with a history of fracture in the same finger, osteoarthritis, or inflammatory arthritis or any history of surgery were excluded. We conducted this study in compliance with the principles of the Declaration of Helsinki. The protocol of this study was reviewed and approved by the Institutional Review Board of Inje University Sanggye Paik Hospital (IRB No. SGPAIK 2018-11-002). Written informed consents were exempted. Of 96 patients (101 fractures), 11 patients could not be followed up; thus, 85 patients ( 88 fractures) were included in this study. The mean age of the 85 patients was 27 years (range, 10 to 86 years), and the patients were predominantly male (61\%). The fifth finger was the most commonly affected digit (47\%), and the other fingers were affected with a similar frequency. Fractures in the nondominant hand were most common (59\%). Hyperextension during sports activities such as playing with a ball (47\%) was the most frequent cause of injury, followed by a fall onto an outstretched hand (38\%). The mean follow-up duration was 23 weeks (range, 12 weeks to 29 months).

The fingers were initially treated with immobilization of the PIP joint in $40^{\circ}$ of flexion by using a dorsal block splint or thermoplastic finger cast, and the joints were gradually extended by $10^{\circ}-15^{\circ}$ per week. After 3 weeks, active PIP joint motion was allowed, and a straight volar splint was used to protect the joint at night. Sports activities were permitted at 6 weeks after immobilization. The minimum time for determining the failure of conservative treatment was 6 weeks. If pain in the end-range motion or limitation of ROM persisted after conservative treatment, excision of the fragment was considered. In the follow-up visit, clinical and radiological outcomes including pain at rest, tenderness, ROM, pain during ROM, ligament instability, fracture union, and subjective satisfaction were evaluated. If the patient complained of limited ROM or pain during ROM with tenderness, delayed excision was recommended. Among 88 fractures, 70 fractures $(80 \%)$ in 67 patients were successfully treated with a finger splint. Eighteen patients (18 fractures) had unsatisfactory outcome after conservative treatment and all agreed to undergo fragment excision after an average of 75 days (range, 35 to 145 days).

For excision of the fragment, a volar Brunner skin incision was performed, and an A3 pulley was divided from the flexor sheath as a lateral flap. The flexor digitorum profundus and superficialis tendons were retracted laterally, and the volar plate was exposed. The avulsed fracture fragment was excised with a sharp scalpel, and the extension of the PIP joint was examined. The remaining volar plate was not sutured, and the wound was closed. Immediate postoperative active ROM exercises were encouraged without any protective device.

A concomitant collateral ligament injury was identified by a survey on tenderness and a stress test. Pain after treatment was categorized as no pain, pain on exertion, pain with activities of daily living, and resting pain. The shape of the fracture fragments was described as a dot, sliver, triangle, or rhomboid on lateral radiographs (Fig. 1). ${ }^{5)}$ Fragment size was evaluated as the area by multiply- 
Lee et al. Conservative Treatment of Volar Plate Avulsion Fractures of Proximal Interphalangeal Joint

Clinics in Orthopedic Surgery • Vol. 12, No. 3, $2020 \bullet$ www.ecios.org

ing the width by the height, both of which were measured on lateral radiographs (Fig. 2A). ${ }^{5}$ The extent of displacement and rotation of the fragment and the status of comminution were assessed (Fig. 2B and C). All radiological measurements were performed by a single hand surgeon (SL).

To identify the factors related to the failure of conservative treatment, we compared the following factors between the cases treated successfully by conservative management (success group: 67 patients, 70 fractures) and the cases treated by operation after conservative treatment (failure group: 18 patients, 18 fractures): age, concomitant dislocation, ROM, pain, and radiological parameters including the size, displacement, and rotation of the fragments. Statistical analyses were performed by using the Student $t$-test, Mann-Whitney test, chi-square test, Fisher exact test, paired $t$-test, Wilcoxon signed-rank test, and multiple logistic regression test.
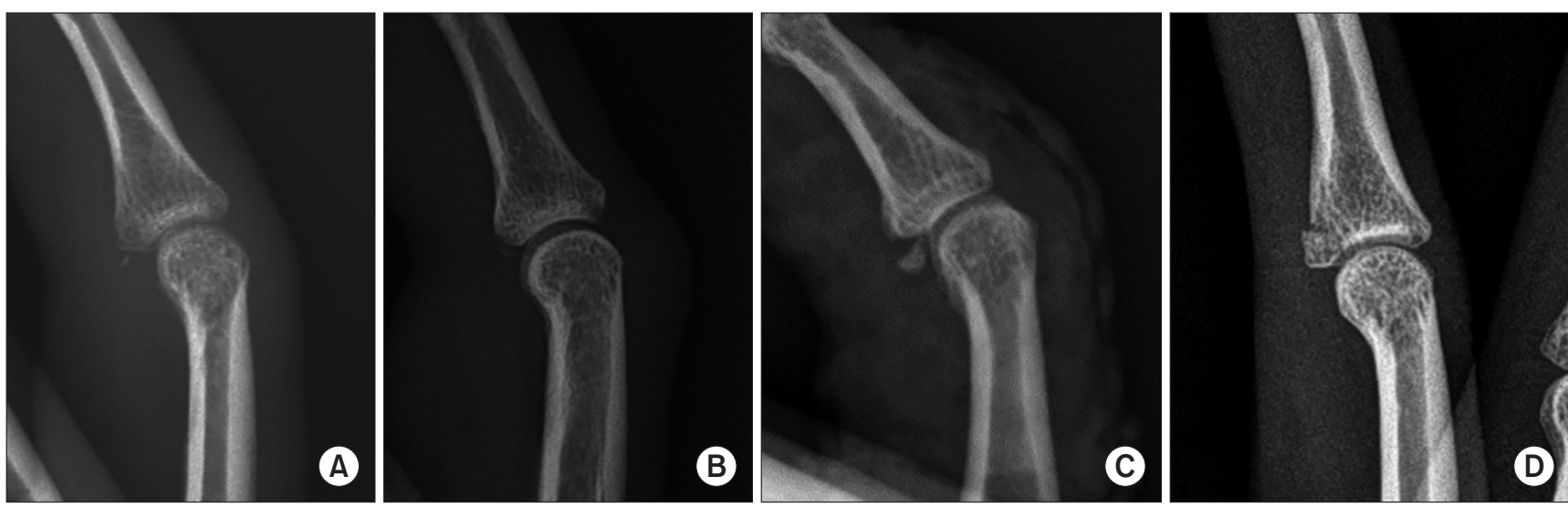

Fig. 1. The shape of the fracture fragment on lateral $X$-rays described as dot $(A)$, sliver $(B)$, triangle $(C)$, or rhomboid (D).
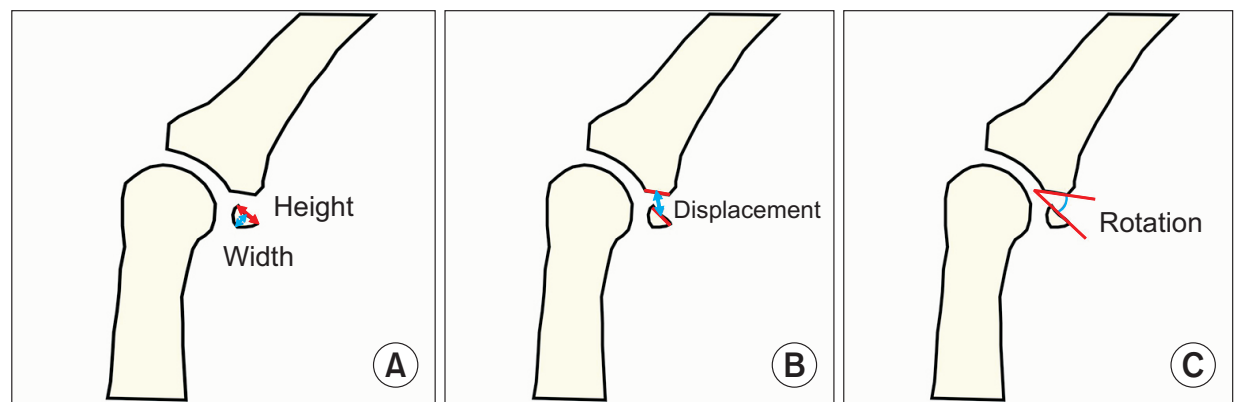

Fig. 2. (A) On the lateral radiograph, the longest proximal-distal length was defined as the height and the longest anteroposterior line perpendicular to it, as the width. The fragment size was evaluated as the area by multiplying the width by the height. (B) The distance between the bisector at the volar fracture surface of the middle phalangeal base and the bisector at the fracture fragment was measured to assess the extent of displacement. (C) The angle created between the volar fracture surface of the middle phalangeal base and the surface of the fracture fragment was measured to assess the extent of rotation. 
Lee et al. Conservative Treatment of Volar Plate Avulsion Fractures of Proximal Interphalangeal Joint

Clinics in Orthopedic Surgery • Vol. 12, No. 3, $2020 \bullet$ www.ecios.org

fingers with collateral ligament injury. The distributions of ligament injury showed no statistically significant difference between the groups $(p=0.461)$.

In the success group (70 fractures), no pain was reported in 58 fractures $(83 \%)$ after conservative treatment, and pain with activities of daily living was noted in 7 fractures. In the failure group (18 fractures), pain with activities of daily living was noted in 13 fractures and resting pain was reported in 4 fractures after conservative treatment (Fig. 3). The distribution of the level of pain after conservative treatment was statistically significantly different between the groups $(p<0.001)$. After operation, no pain was reported in 16 (89\%) of the 18 fractures. The preoperative and postoperative pain distributions were different in this group $(p<0.001)$. In the success group, nonunion was observed in 18 fractures, with 3 cases reporting pain with activities of daily living and 1 case reporting pain on exertion.

After conservative treatment, the mean flexion contracture of the PIP joint was larger, and the mean further flexion and ROM were smaller in the failure group than those in the success group (Table 1). After operation, the mean flexion contracture decreased, and the mean further flexion and ROM increased.

The most common shape of the fracture fragments was triangle (64\% in the success group and $56 \%$ in the failure group), followed by sliver. The distributions of the shapes were not statistically significantly different between the groups ( $p=0.741$ ) (Fig. 4). Fragments were comminuted in $16 \%$ of the conservative cases and in $11 \%$ of the operative cases, showing no statistically significant difference, and the prevalence of fragment comminution was not statistically significantly different $(p>0.99)$.

The mean fragment size, evaluated as the area by

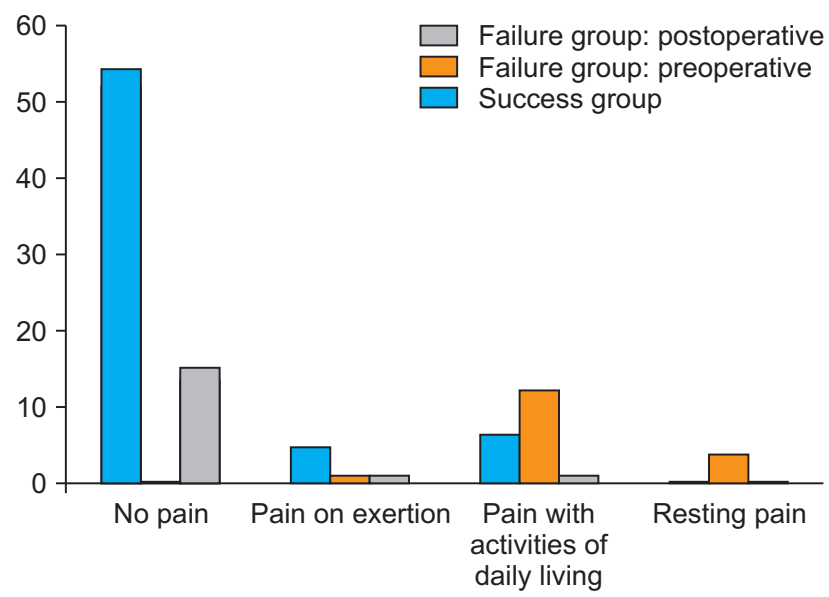

Fig. 3. Distribution of the level of pain in the success and failure groups after conservative treatment. multiplying the width by the height, was similar between the groups (Table 2). The extent of displacement and rotation of fragments was greater in the failure group than in the success group.

We performed univariate regression test for each variable and identified the potential predictors of failure

Table 1. Range of Motion of Each Group after Conservative Treatment and after Operation

\begin{tabular}{|c|c|c|c|c|}
\hline Variable & Average & $\begin{array}{l}\text { Standard } \\
\text { deviation }\end{array}$ & $\begin{array}{c}95 \% \\
\text { Confidence } \\
\text { interval }\end{array}$ & $p$-value \\
\hline \multicolumn{5}{|c|}{ Flexion contracture $\left({ }^{\circ}\right)$} \\
\hline Success group & 4.64 & 7.91 & $2.76-6.52$ & \multirow{2}{*}{$<0.001^{*}$} \\
\hline $\begin{array}{l}\text { Failure group: } \\
\text { preoperative }\end{array}$ & 14.17 & 11.15 & 8.62-19.71 & \\
\hline $\begin{array}{l}\text { Failure group: } \\
\text { postoperative }\end{array}$ & 8.89 & 10.23 & 3.80-13.97 & $0.002^{*}$ \\
\hline \multicolumn{5}{|l|}{ Further flexion $\left({ }^{\circ}\right)$} \\
\hline Success group & 94.71 & 9.55 & $92.44-96.99$ & \multirow{2}{*}{$0.001^{*}$} \\
\hline $\begin{array}{l}\text { Failure group: } \\
\text { preoperative }\end{array}$ & 77.50 & 18.81 & $68.15-86.85$ & \\
\hline $\begin{array}{l}\text { Failure group: } \\
\text { postoperative }\end{array}$ & 91.11 & 9.00 & 86.63-95.59 & $0.017^{*}$ \\
\hline
\end{tabular}

Range of motion $\left({ }^{\circ}\right)$

\begin{tabular}{ccccc} 
Success group & 90.07 & 14.90 & $86.53-93.62$ & \\
$\begin{array}{c}\text { Failure group: } \\
\text { preoperative }\end{array}$ & 63.33 & 24.01 & $51.39-75.27$ & $<0.001^{*}$ \\
$\begin{array}{c}\text { Failure group: } \\
\text { postoperative }\end{array}$ & 82.22 & 15.93 & $74.30-90.14$ & $0.003^{*}$ \\
\hline & & & & \\
\hline
\end{tabular}

${ }^{*} p<0.05$

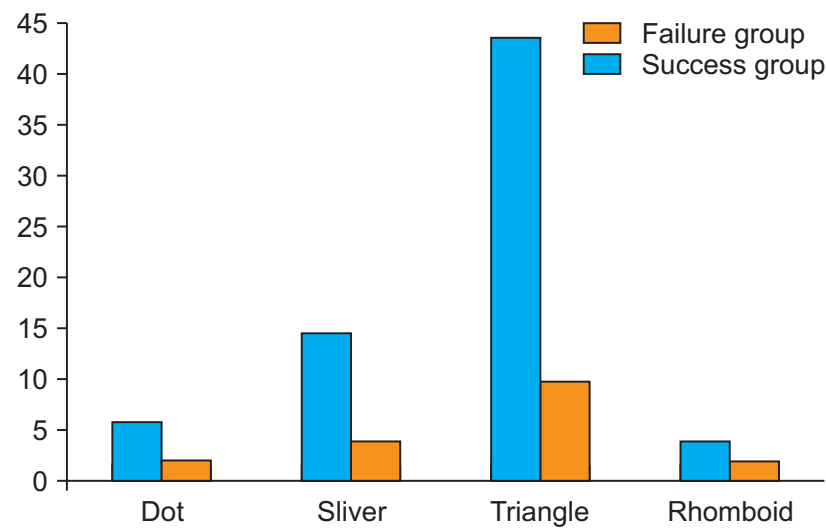

Fig. 4. Distribution of the shape of the fragments in the success and failure groups. 
Lee et al. Conservative Treatment of Volar Plate Avulsion Fractures of Proximal Interphalangeal Joint

Clinics in Orthopedic Surgery • Vol. 12, No. 3, $2020 \bullet$ www.ecios.org

\begin{tabular}{|c|c|c|c|c|}
\hline Variable & Average & $\begin{array}{l}\text { Standard } \\
\text { deviation }\end{array}$ & $\begin{array}{c}95 \% \\
\text { Confidence } \\
\text { interval }\end{array}$ & $p$-value \\
\hline \multicolumn{5}{|l|}{ Fragment size $\left(\mathrm{mm}^{2}\right)$} \\
\hline Success group & 3.51 & 2.72 & $2.86-4.15$ & \multirow{2}{*}{0.698} \\
\hline Failure group & 3.24 & 2.12 & $2.18-4.29$ & \\
\hline \multicolumn{5}{|c|}{ Fragment displacement (mm) } \\
\hline Success group & 0.64 & 0.45 & $0.53-0.74$ & \multirow{2}{*}{$0.002^{*}$} \\
\hline Failure group & 1.51 & 1.01 & $1.01-2.01$ & \\
\hline \multicolumn{5}{|c|}{ Fragment rotation $\left({ }^{\circ}\right)$} \\
\hline Success group & 17.98 & 28.94 & $11.10-24.87$ & \multirow{2}{*}{$<0.001^{*}$} \\
\hline Failure group & 78.09 & 40.66 & 57.87-98.32 & \\
\hline
\end{tabular}

${ }^{*} p<0.05$.

with $p$-values less than 0.05 as follows: displacement, rotation of the fragments, flexion contracture, further flexion, and ROM of the joints. In multiple logistic regression test with these potential predictors, only fragment rotation showed statistical significance ( $p=0.010$ ): larger rotation was more likely to result in failure after conservative treatment (odds ratio, 1.425) (Table 3).

\section{DISCUSSION}

After conservative treatment of volar plate avulsion fractures of the PIP joint, the failure group had a higher prevalence of joint dislocation and greater pain than the success group. The failure group had greater flexion contracture and less further flexion and ROM of the joint after conservative treatment. In this group, the pain and ROM improved after excision of the volar plate avulsion fragments. The shape, comminution, and size of the fracture fragments showed no association with the need for operative treatment, but the failure group had greater fracture displacement and fragment rotation than the success group.

In larger volar plate fractures, the fragments are usually fixated internally with mini screws or pull-out sutures in acute stages, and this method produces favorable results. ${ }^{6}$ Internal fixation of large fragments is considered to be mandatory if the fracture is unstable and accompanied by dislocation. ${ }^{7,8)}$ We have performed conservative treatment using a dorsal block splint if the joint is stable after reduction even if dislocation is predisposed. However, after conservative treatment, operative treatment is recom-
Table 3. Results of Multivariate Logistic Regression Test

\begin{tabular}{lccccc}
\multicolumn{1}{c}{ Variable } & B & $\begin{array}{c}\text { Standard } \\
\text { error }\end{array}$ & $p$-value & Exp (B) $\begin{array}{c}\text { Confidence } \\
\text { interval for } \\
\text { exp (B) }\end{array}$ \\
\hline $\begin{array}{l}\text { Fragment } \\
\text { displacement }\end{array}$ & 0.354 & 0.542 & 0.513 & 1.425 & $0.492-4.127$ \\
$\begin{array}{l}\text { Fragment rotation } \\
\text { Froxion }\end{array}$ & 0.025 & 0.009 & $0.010^{*}$ & 1.025 & $1.006-1.044$ \\
$\begin{array}{l}\text { Flexion } \\
\text { contracture }\end{array}$ & 0.020 & 0.040 & 0.618 & 1.020 & $0.943-1.103$ \\
Further flexion & -0.060 & 0.032 & 0.062 & 0.942 & $0.855-1.003$ \\
Constant & 2.244 & 3.010 & 0.456 & 9.436 & - \\
\hline${ }^{*} p<0.05$. & & & & &
\end{tabular}

mended for patients with unfavorable results in terms of pain and ROM.

The "conservative treatment first" method has some advantages. After the conservative treatment period, the volar plate fractures in the failure group were found to be in a subacute or chronic status, the collateral ligaments were healed, and even the initially unstable joint was stabilized. Therefore, compared to those used in acute cases, the techniques required for surgical treatment were relatively simple; specifically, only excision of the fracture fragments was considered necessary. In the acute cases, if the fragments are large, fixation rather than excision may need to be performed for stability of the joint, and sometimes, volar plate arthroplasty is necessary to avoid dorsal subluxation of the joint. ${ }^{9)}$ If the fracture is concomitant with collateral ligament rupture, repair of the ligaments should be considered even when the fractures are fixated. ${ }^{10}$ ) After conservative treatment, the joint became stable or rather stiff, without possibility of instability after excision of the fragments. The box complex that comprises the proper and accessory collateral ligaments and volar plate encloses the PIP joint volarly and laterally and prevents hyperextension of the joint after fragment excision. Therefore, immediate ROM exercises after operation were possible, and a splint or brace was not necessary to protect the joint. Moreover, excision of the volar plate fragments released the flexion contracture of the joint to loosen the checkrein ligaments and improved the joint ROM.

The average size of the fragments in the failure group was $3.24 \mathrm{~mm}^{2}$ on lateral radiographs and was too small to be fixated with screws. If an open reduction is performed with Kirschner wires, sutures, or pull-out wires, the joint should be immobilized until union is achieved and the joint motion would be limited after immobilization. Therefore, the best operative choice in our cases was 
Lee et al. Conservative Treatment of Volar Plate Avulsion Fractures of Proximal Interphalangeal Joint

Clinics in Orthopedic Surgery • Vol. 12, No. 3, $2020 \bullet$ www.ecios.org

delayed excision.

In our study, $20 \%$ of the volar plate fractures were eventually treated with fragment excision after conservative treatment and had a higher prevalence of concomitant joint dislocation at the time of injury and greater fracture displacement and fragment rotation. The risk factors for operative treatment seemed to be concomitant joint dislocation at the time of injury and larger fracture displacement and fragment rotation. As per the $95 \%$ confidence intervals of the fragment displacement and rotation presented in Table 2, if patients have volar plate fractures with a history of or displacement of more than $1 \mathrm{~mm}$ or fragment rotation of more than $55^{\circ}$, the possibility of operative treatment after conservative treatment will increase. As delayed excision has many advantages over an immediate operation, we recommend conservative treatment for almost all volar plate avulsion fractures of stable PIP joints.

The failure group complained of more pain and smaller ROM with most pain reported at the end ROM and had improvement in pain after excision of the fragment. We conjecture that the fracture fragment with articular cartilage causes mechanical impingement of the joint, interfering with full ROM and resulting in joint pain. The actual size of a fracture fragment was bigger than that observed on radiographs because cartilaginous components were not visible on radiographs. Moreover, all operative cases had nonunion of an avulsed fragment; nonunion might have also been the cause of pain.

This study has some limitations. First, we assigned the operative cases to the failure group because the patients complaining of limited ROM or pain in ROM with tenderness were recommended operative treatment and all agreed to undergo surgery. However, such surgical indications are based on subjective symptoms and the definite distinguishable indications or methods have not been established. Second, we could not evaluate collateral ligament ruptures because ultrasonography or magnetic resonance imaging of the joint was not performed initially; only tenderness over the collateral ligament and lateral stability were examined. The joint was immobilized in a finger splint or a cast during the initial conservative period. The collateral ligaments might have healed, and none of the cases had residual lateral instability after conservative treatment. Therefore, accurate evaluation of the extent of the collateral ligament rupture was not necessary in this study. Third, the short follow-up period and small sample size were not sufficient to allow definite conclusions. Although almost all cases with good clinical results at 4 to 6 weeks after injury had good or better results after more than 6 months, cases with a relatively short-term followup were also included in our study. Fourth, many patients were not evaluated objectively using the scoring system, so the scoring data could not be analyzed.

In conclusion, the history of joint dislocation and fracture displacement and fragment rotation might be the risk factors for failure of conservative treatment of volar plate avulsion fractures of the PIP joint.

\section{CONFLICT OF INTEREST}

No potential conflict of interest relevant to this article was reported.

\section{ACKNOWLEDGEMENTS}

This work was supported by Basic Science Research Program through the National Research Foundation of Korea funded by the Ministry of Education, Science and Technology of South Korea (NRF-2016R1A2B1013761).

We would like to thank Hyung-Yung Lee (Department of Statistics, Pusan National University, Busan, Korea) for valuable statistical advice.

\section{REFERENCES}

1. Gaine WJ, Beardsmore J, Fahmy N. Early active mobilisation of volar plate avulsion fractures. Injury. 1998;29(8):58991.

2. Phair IC, Quinton DN, Allen MJ. The conservative management of volar avulsion fractures of the P.I.P. joint. J Hand Surg Br. 1989;14(2):168-70.

3. Adi M, Hidalgo Diaz JJ, et al. Results of conservative treatment of volar plate sprains of the proximal interphalangeal joint with and without avulsion fracture. Hand Surg Rehabil. 2017;36(1):44-7.
4. Takami H, Takahashi S, Ando M. Large volar plate avulsion fracture of the base of the middle phalanx with rotational displacement: a report of three cases. J Hand Surg Am. 1997;22(4):592-5.

5. Lee S, Jung EY, Kim JY. Operative treatment for volar plate avulsion fractures of the fingers. Arch Orthop Trauma Surg. 2013;133(10):1463-7.

6. Lee $\mathrm{CH}$, Kim CU, Cha K. Pullout suture using polyamide monofilaments for volar plate avulsion fractures of the proximal interphalangeal joint. J Hand Surg Eur Vol. 
Lee et al. Conservative Treatment of Volar Plate Avulsion Fractures of Proximal Interphalangeal Joint

Clinics in Orthopedic Surgery • Vol. 12, No. 3, $2020 \bullet$ www.ecios.org

2018;43(5):558-60.

7. Chew WY, Cheah AE. Volar plate and screw fixation for dorsal fracture-dislocation of the proximal interphalangeal joint: case report. J Hand Surg Am. 2010;35(6):928-30.

8. Hamilton SC, Stern PJ, Fassler PR, Kiefhaber TR. Miniscrew fixation for the treatment of proximal interphalangeal joint dorsal fracture-dislocations. J Hand Surg Am. 2006;31(8):1349-54.
9. Ng CY, Oliver CW. Fractures of the proximal interphalangeal joints of the fingers. J Bone Joint Surg Br. 2009;91(6):70512.

10. Kim YW, Roh SY, Kim JS, Lee DC, Lee KJ. Volar plate avulsion fracture alone or concomitant with collateral ligament rupture of the proximal interphalangeal joint: a comparison of surgical outcomes. Arch Plast Surg. 2018;45(5):458-65. 